\title{
Robust background subtraction using geodesic active contours in ICA subspace for video surveillance applications
}

\author{
Hicham Sekkati \\ University of Ottawa, VIVA Research LAB \\ Ottawa, Canada \\ Email:sekkati@gmail.com \\ Amar Mitiche \\ Institut National de Recheche Scientifique \\ Montreal, Canada \\ Email: mitiche@emt.inrs.ca
}

\author{
Robert Laganière \\ University of Ottawa, VIVA Research LAB \\ Ottawa, Canada \\ Email: laganier@eecs.uottawa.ca
}

\author{
Richard Youmaran \\ Solink Corporation \\ Ottawa, Canada \\ Email: ryoumaran@solinkcorp.com
}

\begin{abstract}
Current background subtraction methods require background modeling to handle dynamic backgrounds. The purpose of our study is to investigate a background template substraction method to detect foreground objects in the presence of background variations. The method uses a single reference image but the change detection process allows change in the background including illumination changes and dynamic scenes. Using indoor and outdoor scenes, we compare our method to the best state-of-the art algorithms using both quantitative and qualitative evaluation. The results show that our method is in general more accurate and more effective.
\end{abstract}

Keywords-background substraction; detection change; independent component analysis; segmentation; geodesic active contours; level sets;

\section{INTRODUCTION}

Important applications such as surveillance, low-band telecommunications, animation and video event analysis require moving object detection. When the camera acquiring the images is static, background substraction is often used. There are numerous papers on the subject and also several reviews [1], [2]. The simplest scheme to detect the foreground moving objects in an image sequence is to use a fixed background template which is substracted from the current image. The resulting difference image is then analyzed, thresholded, for instance, to isolate the foreground. Background template substraction can be effective with short video sequences in constraint environments, such as indoors, but it is not applicable with a changing background. Several methods have addressed background variations by representing the image at each pixel by Gaussian distribution or a mixture of Gaussians (MoG) learned over a time interval [3], [4], [5], [6], [7]. To improve the MoG model some methods have used color model space [8], Markov Random Fields [9], or non parametric Kernel distributions [10], [11]. Other methods encode pixel changes in the spatiotemporal space [12], [13], [14], [15], [16], [17], [18], [19], while other schemes account explicitly for conditions such as illumination changes [20], non-stationary camera [21], or non-stationary background textures [22]. All of these methods require sensing for a period of time to estimate the model parameters, which must then be continually updated. In general, the update rules are sensitive to sudden illumination changes and uncommon object activities. Such situations often occur in video surveillance applications. In our application, the objective is to monitor an Automated Teller Machine (ATM) environment to assist subsequently the detection of suspicious user activities and abnormal behaviour.

The purpose of our study is to investigate a background template substraction method to detect the foreground of moving objects in the presence of background variations. The method uses a single image but the change detection process allows change in the background including illumination changes and dynamic scenes. This single template is any image acquired when there were no foreground objects. The scheme consists of two steps (1) an ICA decomposition of the current image augmented by the template to form a single signal, giving two images in ICA subspace, one corresponding to the foreground of the moving objects and the other to its complement and, (2) a geodesic curve evolution in the foreground ICA image to detect the moving objects. This leads to an efficient and effective algorithm because the ICA foreground image is devoid of irrelevant regions such as those due to noise and artifacts, allowing the geodesic to better adhere to the foreground boundary. As a result the geodesic curve will move to coincide with the sharp boundary of the foreground objects. Experiments have shown that this does not happen when successive image differencing techniques are used along with geodesic curve evolution. For instance, Figure 2 (rows 3 and 4) shows that the ICA foreground image preserves better the boundary of foreground objects than in the difference image (e.g., in the middle images the person's arm was assigned to the background by differencing images contrary to ICA image 
where the edges between the arm and background are more preserved).

This paper is organized as follows. Section 2 introduces the ICA model. Section 3 presents the geodesic model applied in ICA space. In Section 4 we provide details about the method implementation. Section 5 discusses experimental results including comparisons with other state-of-the-art algorithms. Section 6 concludes the paper.

\section{ICA MODEL}

Independent Component Analysis (ICA) is a well-known technique in signal processing for estimating unknown signals from their observed mixtures [23]. By definition it assumes that, the mixture components are statistically independent. In the case of the background substraction problem this assumption holds for static backgrounds but is not directly applicable to dynamic backgrounds. There has been some work with background substraction using ICA [24]. However the method is only applicable to dynamic image changes due to illumination variations. In the case of dynamic background, background changes can be due to scene illumination variations, a part of the foreground texture is similar to the background texture, moving parts appearing in the background (i.e., leaves, tree branches), highly dynamic background like water surface, etc. When dealing with $2 \mathrm{D}$ images, it is possible to assess the consistency of the extracted ICA signals using the underlying edge and boundary information of the moving objects.

The ICA problem can be stated as the separation of independent sources from an array of sensors which is a classical problem in signal processing [25]. It was solved efficiently using FastICA algorithm [23]. Given n-dimensional random vector $\mathbf{x}=\left[\mathrm{x}_{1}, \cdots, \mathrm{x}_{n}\right]^{T}$ representing the mixing signals measured from $n$ sensors, and $\mathbf{s}=\left[s_{1}, \cdots, s_{n}\right]^{T}$ is $\mathbf{n}$ dimensional random vector representing the independent components signals which are unknown, the problem can be stated as follows:

$$
\mathbf{x}=\mathbf{A} \mathbf{s}
$$

where the $n \times n$ mixing matrix $\mathbf{A}$ should be estimated from only the mixing signals. The independent components can then be derived from the inverse of $\mathbf{A}$ called demixing matrix $\mathbf{W}=\left[\mathbf{w}_{1}, \cdots, \mathbf{w}_{n}\right]^{T}=\mathbf{A}^{-1}$ simply by

$$
\mathbf{s}=\mathbf{W} \mathbf{x}
$$

The FastICA algorithm is based on a fixed-point iteration scheme for finding directions, i.e., unit vectors $\mathbf{w}_{k}$ such that the projection $\mathbf{w}_{k}^{T} \mathbf{x}$ maximizes nongaussianity all by insuring decorelations between them.

For the background substraction problem, let us denote by $I_{1}$ and $I_{2}$ the images of the background scene and the current video frame to be processed respectively. Let us define two new images $I_{f}$ and $I_{b}$ in ICA subspace and representing respectively the foreground defined by the moving objects in the scene, and the background scene defined by the non moving objects. Assuming independence between $I_{f}$ and $I_{b}$, these images can be expressed in term of $I_{1}$ and $I_{2}$ as follows:

$$
\left[\begin{array}{c}
I_{f} \\
I_{b}
\end{array}\right]=\left[\begin{array}{ll}
w_{11} & w_{12} \\
w_{21} & w_{22}
\end{array}\right]\left[\begin{array}{l}
I_{1} \\
I_{2}
\end{array}\right]
$$

where the coefficients $w_{i j}$ are the elements of the $2 \times 2$ matrix $\mathbf{W}$. In the case of a static background the matrix $\mathbf{W}$ takes a simple form; in the ideal case it can be expressed by:

$$
w_{11}=-w_{12}, \quad w_{21}=1, \quad w_{22}=0 ;
$$

hence allowing to extract the foreground by simply substracting images $I_{1}$ and $I_{2}$. For the case of a dynamic background, the components $I_{f}$ and $I_{b}$ are no longer independent which makes it impossible to separate the correlated components of the signal using only equation 3 . It will be shown in the experiments that a naive thresholding on $I_{f}$ or even using more elaborated model on image space will fail to segment the moving objects from the background scene.

\section{Segmentation in ICA subspace}

A robust and accurate segmentation technique is then required to segment the image $I_{f}$ and that allow capturing in ICA subspace the edges of the moving objects. The image $I_{f}$ is characterized by high transitions at the location of moving objects and can be efficiently segmented using boundarybased segmentation schemes. Evolving interfaces (curves) according to some flow is becoming a popular technique to address boundary-based segmentation. Here we use the general model of the geodesic active contours (GAC) that offers mainly three advantages [26]: a) The model can be represented by the level sets formalism, thereby topological changes are naturally handled [27]; b) also it does not require a prior knowledge of the number of moving objects therefore it can detect the boundaries of an arbitrary number of moving objects; c) the model does not require many parameters to weight the contributions of different terms unlike most of the proposed edge-based segmentation methods.

Applying the general model of GAC in ICA space will minimize the following intrinsic edge-based functional:

$$
\begin{aligned}
E(\gamma(s))= & \int_{\gamma} g\left(\left\|\nabla I_{f}(\gamma(s))\right\|\right)\|\dot{\gamma}(s)\| d s+ \\
& \lambda \int_{\Omega_{\gamma}} g\left(\left\|\nabla I_{f}(x, y)\right\|\right) \mathrm{d} x \mathrm{~d} y
\end{aligned}
$$

Where $\gamma(s)$ is a planar closed curve, $\Omega_{\gamma}$ is the interior region of the curve $\gamma$, and $g$ is a positive monotonically decreasing edge indicator function. A common choice of $g$ is $g(u)=\frac{1}{1+u^{2}}$. The first term in (4) is an image dependent force guided by the stopping function $g$ with the main goal 
to stop the evolving curve when it arrives to the objects boundaries. The weight $\|\dot{\gamma}(s)\|$ allow the first term to be invariant to parametrization of the curve $\gamma$. The second term in (4), weighed by the the real number $\lambda$, mainly allows the detection of non-convex objects and is also of importance to increase the speed of convergence [26].

Finding the minimum of (4) by steepest-descent method is equivalent to solve the associated Euler-Lagrange equations which lead to the following curve evolution equation:

$\frac{\partial \gamma(s, t)}{\partial t}=\left[g\left(\left\|\nabla I_{f}\right\|\right) \kappa-\nabla g\left(\left\|\nabla I_{f}\right\|\right) \cdot \mathbf{n}+\lambda g\left(\left\|\nabla I_{f}\right\|\right)\right] \mathbf{n}$

where $\mathrm{t}$ denotes the time as the curve evolves, $\kappa$ is the curvature of $\gamma$, and $\mathbf{n}$ is the inward Euclidean normal vector to the curve $\gamma(s, t)$.

Using the level sets representation, the curve $\gamma$ can be represented by the zero level set of two dimensional function $\phi(u, t)$. Assuming that the embedding level sets function takes negative values inside the zero level contour and positive values outside, the inward normal vector and the curvature can be expressed as

$$
\mathbf{n}=-\frac{\nabla \phi}{\|\nabla \phi\|}, \quad \kappa=-\operatorname{div}(\mathbf{n},)
$$

and the embedding function, $\phi$, should deforms according to the following evolution equation:

$$
\begin{aligned}
\frac{\partial \phi(u, t)}{\partial t}= & g\left(\left\|\nabla I_{f}\right\|\right) \kappa\|\nabla \phi\|+\nabla g\left(\left\|\nabla I_{f}\right\|\right) \cdot \nabla \phi+ \\
& \lambda g\left(\left\|\nabla I_{f}\right\|\right)\|\nabla \phi\|
\end{aligned}
$$

In the experiments, the results from the GAC-ICA model in (4) will be compared to the geodesic active contour model applied to the difference frames in image space by replacing $I_{f}$ with $I=\left|I_{1}-I_{2}\right|$. This will show by evidence that edges in ICA space are more tractable than in image space.

\section{IMPLEMENTATION}

A direct implementation of the evolution equation (7) is computationally expensive as it relies on computing the evolution of all the level sets, not simply the zero level set corresponding to the curve itself. In order to evolve efficiently the level sets, two different implementations have been proposed, the Narrow Band and the Fast Marching algorithms [27]. The Fast Marching algorithm considers only the special case of moving the interface with a monotonically advancing speed. In the Narrow band implementation, the speed by which the front move can be of any sign and a set of narrow band pixels is defined around the latest front position and the level set function is updated only within this band. We use the narrow band implementation of ITK package by choosing the right parameters to accommodate the general geodesic active contour model. This implementation allows weighting three terms in the propagation speed according to the following level sets evolution equation:

$$
\frac{\partial \phi(u, t)}{\partial t}=\gamma Z(u) \kappa\|\nabla \phi\|+\alpha A(u) \cdot \nabla \phi+\beta P(u)\|\nabla \phi\|
$$

where $Z(u), A(u)$, and $P(u)$ are three spatial functions weighted respectively by three scalars $\gamma, \alpha$, and $\beta$. To accommodate the implementation in Caselles's paper, we set all the spatial functions to the edge indicator function i.e., $A(u)=Z(u)=P(u)=g(u)$, and the parameters are set to $\gamma=\alpha=1$, and $\beta=\lambda$. In all our experiments, we initialize the curve near the image boundaries. The parameter $\lambda$ weighting the balloon force is set to positive values within the interval $[0.23 .5]$ depending on edges strength in ICA images.

\section{EXPERIMENTS}

We conducted two sets of experiments. One is to compare our method to the methods reported in the survey of [1]. The other consists of two subsets of experiments. One is to compare our method to ViBe [12] and Zivkovic-GMM [6] which are state-of-the-art methods [2]. The other compares the proposed method to $G A C$ acting on a simple difference image. This second set of experiment is to show the relevance of working in the ICA subspace rather than on the image space.

\section{A. Qualitative analysis}

We tested the proposed model on many indoor and outdoor natural sequences with dynamic backgrounds and/or illumination changes. The sequences were selected from different datasets benchmarks as well as from real ATM cameras to deal with varying aspects of the difficulties that can be encountered in many real scenarios. Initialization of the level sets is shown as the zero-level set on the top of ICA image, and also shown the final segmentation obtained by evolving the level set according to the PDE in equation (7). The binary masks obtained with our model GAC-ICA are compared with the ground-truth, ViBe and Zivkovic-GMM methods. The first sequence, called Street and selected from [18], involves minimal motion of the camera with swaying trees in the background. Frames 1 and 389 are the two input images used for this sequence. The second sequence, called Bottle from [22], captures a flowing river with floating bottle. The first frame of the waving river sequence together with the frame 36 of the floating bottle sequence are used. The segmentation results of both sequences are shown in Figure 1. Those results show that the proposed model performs within the same level of methods relying on temporal information while ours using only two images. Three other sequences from our ATM dataset were selected to show the robustness of the proposed model. The first sequence that we call ATM1 
1
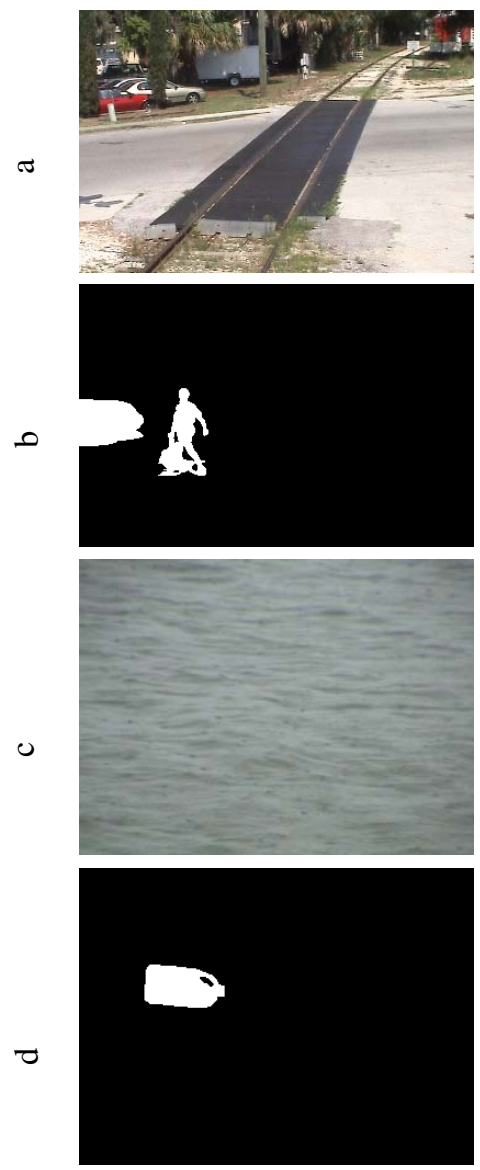

2
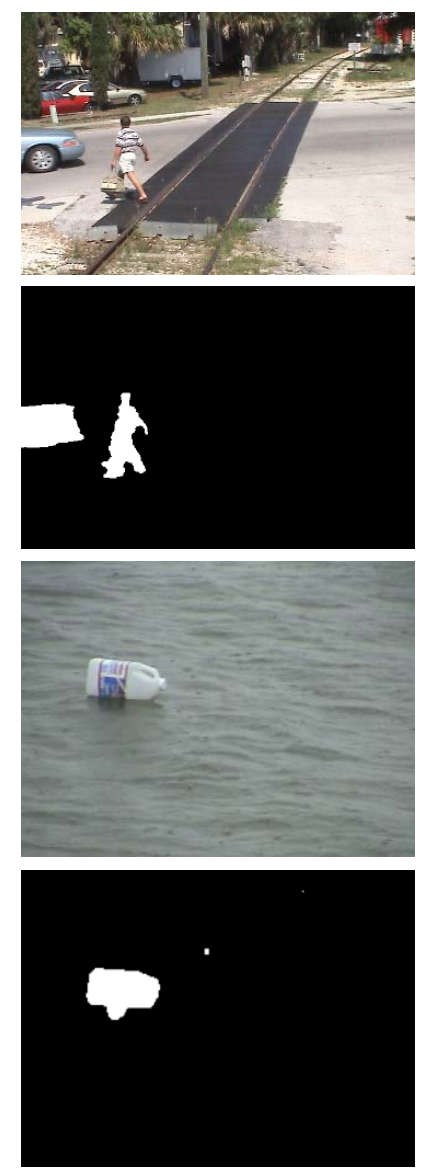

3
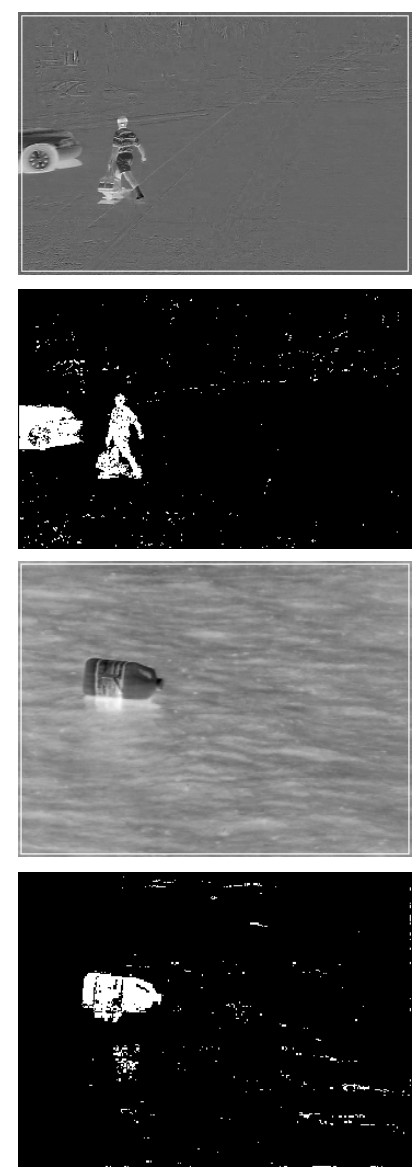

4
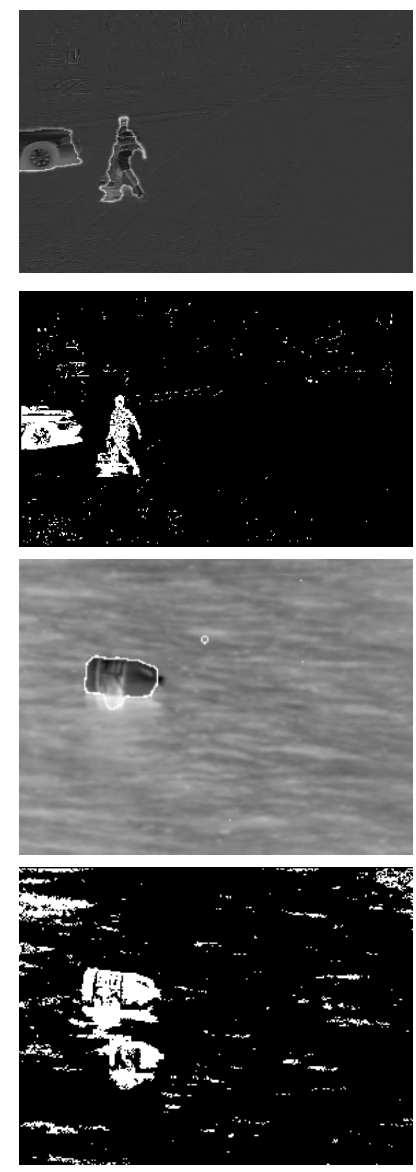

Figure 1. The pair of images used in the sequence Street (resp. Bottle) are shown in (a1, a2) (resp. (c1, c2)). Initialization of the zero level sets on the top of ICA image is shown in a3 (resp. c3), and final segmentation obtained by evolving the level set according to the PDE in equation (7) is displayed in a4 (resp. c4). The ground-truth binary mask is shown in b1 (resp. d1), the mask obtained with our model GAC-ICA is shown in b2 (resp. d2), and the masks from the method ViBe b3 (resp. d3) and from the method Zivkovic-GMM b4 (resp. d4) are shown for comparison.

capture a person casting a shadow in front an ATM and his clothing is highly camouflaged in the background scene. The second sequence called ATM2 captures a person doing a transaction in front an ATM. There is a significant change of illumination in the whole scene with sudden change on the screen of the ATM and the door. Also a shadow of a person appears on the glass while opening slightly the door. The third sequence Fridge capture a person in cluttered and dynamic environment. The camera has moved slightly and many objects have changed their positions. The results of those three sequences are displayed in the Figure 2. To show the advantage of segmentation in ICA space, we compare the results from our GAC-ICA model with the same model applied to the image difference. We compare also the results of our method with an Otsu thresholding on ICA images. The results show that ICA model is not appropriate to extract background for scenes with sudden illumination changes or dynamic background. Another comparison with the linear dependence detector $L D D$ proposed in [28] shows that, even using statistics on image space that are invariant to illumination changes, our method shows better effectiveness.

\begin{tabular}{l|c|c|c|c|c|c|c|}
\hline Sequence & MO & TD & LS & WT & C & B & FA \\
\hline TE $=$ FP+FN & 0 & 415 & 591 & 450 & 800 & 1769 & 439 \\
\hline
\end{tabular}

Table I

ERRORS OBTAINED USING OUR METHOD GAC-ICA FOR THE SEVEN SEQUENCES OF Wallflower DATASET; THE TOTAL ERROR IS COMPUTED AS A SUM OF PIXELS THAT ARE MISS ASSIGNED TO BACKGROUND AND FOREGROUND COMPARING TO GROUND TRUTH. FP: THE NUMBER OF

FALSE POSITIVES THAT COUNTS THE NUMBER OF BACKGROUND PIXELS INCORRECTLY CLASSIFIED AS FOREGROUND; FN: THE NUMBER OF FALSE NEGATIVES WHICH ACCOUNTS FOR THE NUMBER OF FOREGROUND PIXELS INCORRECTLY CLASSIFIED AS BACKGROUND.

\section{B. Quantitative analysis}

To assess the performance of the proposed method $G A C$ $I C A$, we use the Wallflower dataset [29] and we compare our model with other methods for which the results were re- 

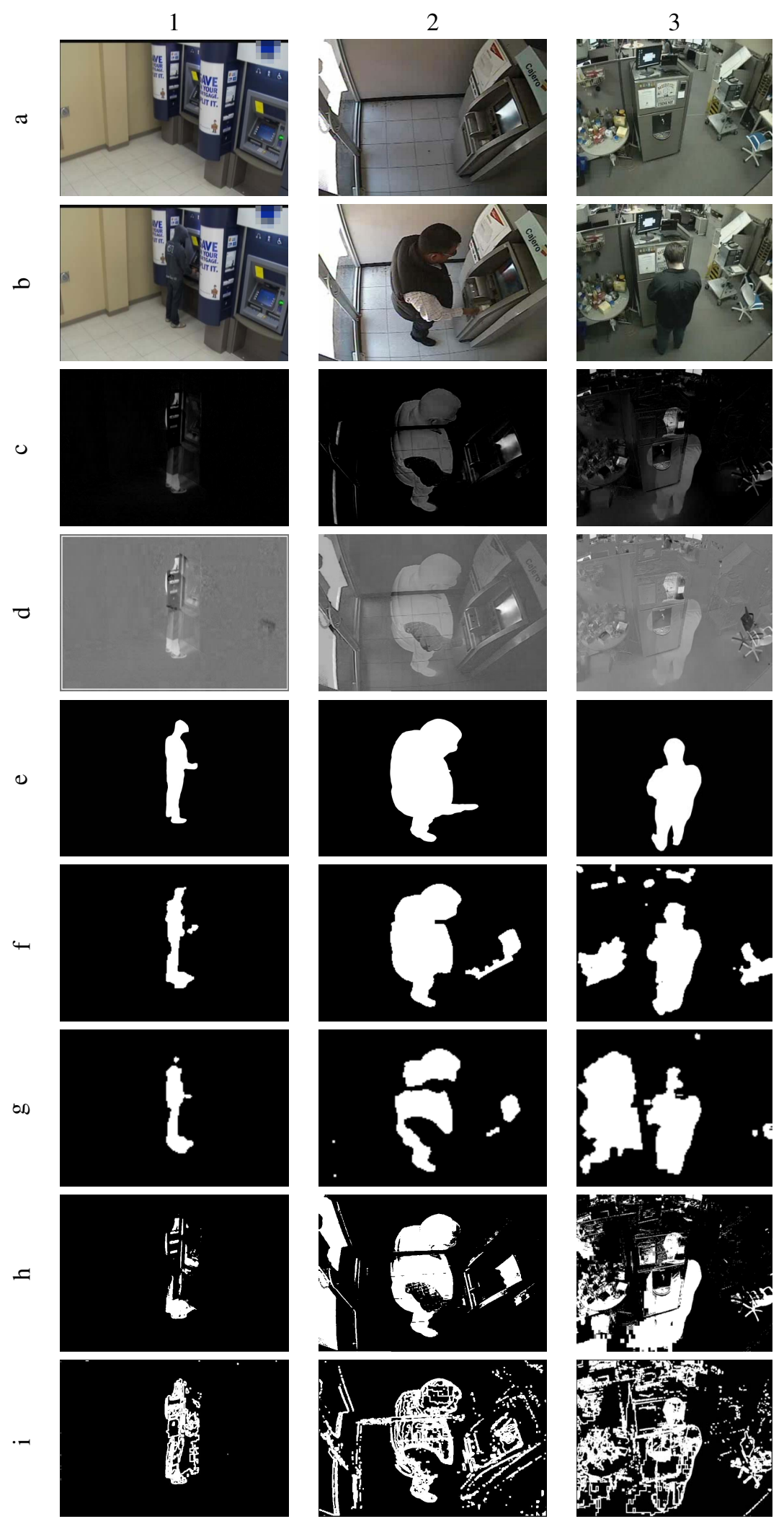

Figure 2. The pair of images used for the sequence ATM1 (resp. ATM2 and Fridge) are shown in (a1, b1) (resp. (a2, b2) and (a3, b3)). The difference of frames is shown in $\mathrm{c} 1$ (resp. $\mathrm{c} 2$ and $\mathrm{c} 3$ ), and the foreground ICA image is shown in $\mathrm{d} 1$ (resp. $\mathrm{d} 2$ and $\mathrm{d} 3$ ). The ground-truth binary mask is shown in e1 (resp. e2 and e3), segmentation with the model GAC-ICA is shown in f1 (resp. f2 and f3), segmentation with the model GAC-DDF is shown in $\mathrm{g} 1$ (resp. $\mathrm{g} 2$ and g3), Otsu thresholding ICA image is displayed in h1 (resp. h2 and h3), and substraction with $L D D$ method is shown in i1 (resp. i2 and i3). 


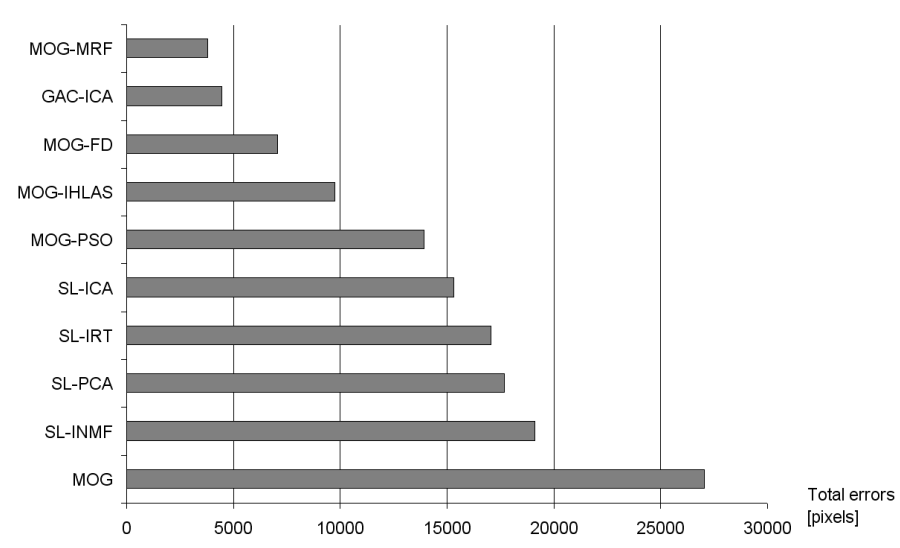

Figure 3. Overall performance on the Wallflower dataset using nine methods from [1] and compared to GAC-ICA.
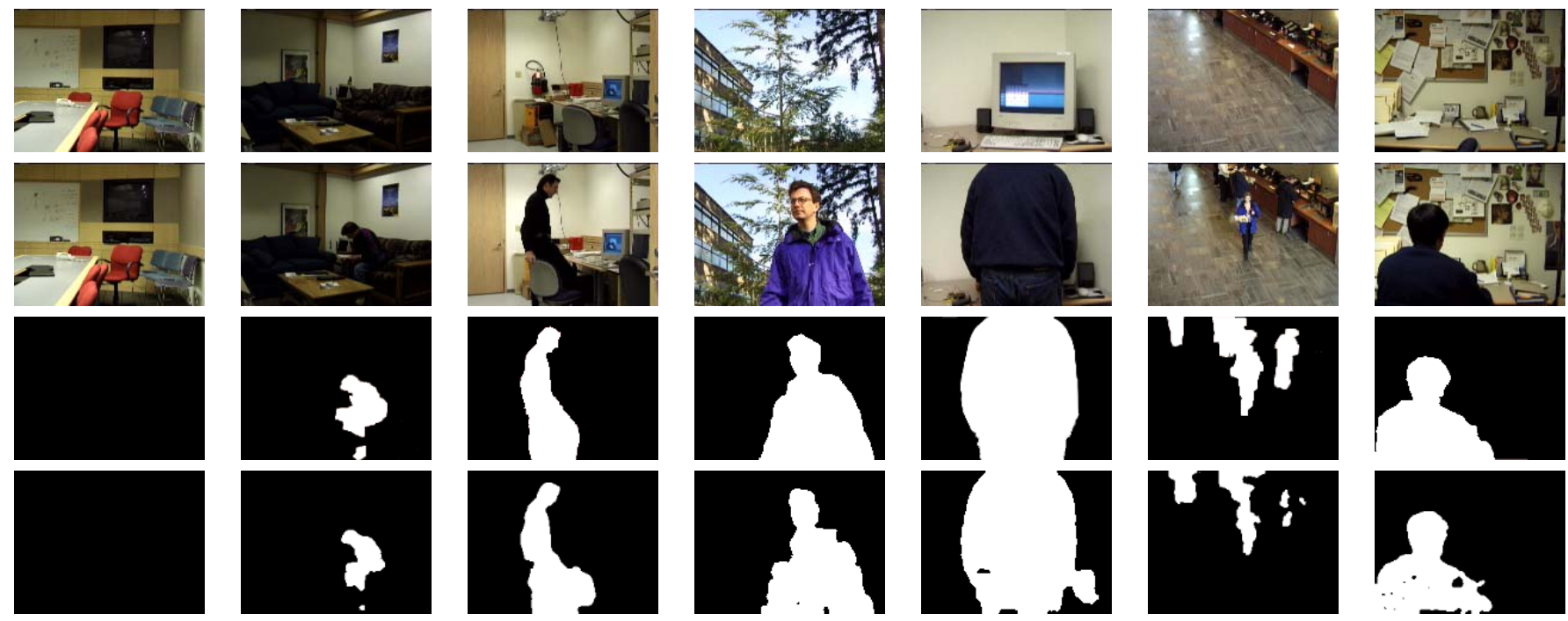

Figure 4. The first and second lines show the pair of images used for the Wallflower dataset. The ground-truth binary mask and the segmentation with our model GAC-ICA are shown respectively in lines 3 and 4.

ported in the survey [1]. There are nine methods denoted by $M O G[4], M O G-P S O[30], M O G-I H L S[31], M O G-F D[32]$, $M O G-M R F$ [9], SL-PCA[33], SL-ICA[24], SL-INMF [34], SLIRT [35]. Note that all those methods use the temporal information to model the background, except for the method $S L$ $I C A$. The dataset consists of seven video sequences in which each sequence presenting one or more of the challenges that may be present in dynamic background and change illuminations scenes. A brief description of those sequences is also given in the survey [1] and we will refer to them with the same notations i.e., Moved Object (MO), Time of Day (TD), Light Switch (LS), Waving trees (WT), Camouflage (C), Bootstrapping (B), and Foreground Aperture (FA). For each sequence, we use a frame of the reference background (an empty scene) and the frame for which the ground truth mask was provided. The results using the GAC-ICA model are shown in figure 4 . The errors are measured as the total of misclassified pixels with respect to ground truth that is $\mathrm{TE}=\mathrm{FP}+\mathrm{FN}$. Table (I) shows the obtained errors by applying our model to the seven sequences of Wallflower and in figure 3 we compare them to the errors obtained from the nine methods.

\section{CONCLUSION}

We proposed a Background Subtraction method whose effectiveness was demonstrated to successfully cope with dynamic background and illumination changes in the scene. The model uses decomposition of images in ICA space followed by edge-based segmentation using geodesic active contour model. Experimental results showed that the geodesic curve adheres better to the boundary of the moving objects in ICA space than in image difference. We compared the proposed method with the best state-of-the arts techniques for background subtraction algorithms and showed that the method has a high precision and detection rate in 
comparison with other methods. An extension of this work using more than two images in ICA space combined with edge-based multiregion segmentation would be useful for tracking moving objects within a scene.

\section{ACKNOWLEDGMENT}

This work was supported by the NSERC under the Engage program and done in collaboration with Solink corporation.

\section{REFERENCES}

[1] T. Bouwmans, "Recent advanced statistical background modeling for foreground detection - a systematic survey," Recent Patents on Computer Science, vol. 4, no. 3, pp. 147-176, 2011.

[2] S. Brutzer, B. Höferlin, and G. Heidemann, "Evaluation of background subtraction techniques for video surveillance," in CVPR. IEEE, 2011, pp. 1937-1944.

[3] C. Wren, A. Azarbayejani, T. Darrell, and A. Pentland, "Pfinder: Real-time tracking of the human body," IEEE Transactions on Pattern Analysis and Machine Intelligence, vol. 19, pp. 780-785, 1997.

[4] C. Stauffer and W. E. L. Grimson, "Adaptive background mixture models for real-time tracking," in CVPR, 1999, pp. II: $246-252$.

[5] N. Friedman and S. Russell, "Image segmentation in video sequences: A probabilistic approach," in Proceedings of the 13th Conference on Uncertainty in Artificial Intelligence (UAI-97), D. Geiger and P. P. Shenoy, Eds. San Francisco: Morgan Kaufmann Publishers, Aug. 1-3 1997, pp. 175-181.

[6] Z. Zivkovic and F. van der Heijden, "Efficient adaptive density estimation per image pixel for the task of background subtraction," Pattern Recognition Letters, vol. 27, no. 7, pp. 773-780, May 2006.

[7] D.-S. Lee, "Effective gaussian mixture learning for video background subtraction," IEEE Transactions on Pattern Analysis and Machine Intelligence, vol. 27, no. 5, pp. 827-832, 2005.

[8] P. Kaewtrakulpong and R. Bowden. (2001, Sep.) An Improved Adaptive Background Mixture Model for Realtime Tracking with Shadow Detection.

[9] K. Schindler and H. Z. Wang, "Smooth foregroundbackground segmentation for video processing," in $A C C V$, 2006, pp. II:581-590.

[10] A. M. Elgammal, D. Harwood, and L. S. Davis, "Nonparametric model for background subtraction," in ECCV, 2000, pp. II: 751-767.

[11] C. Ianaşi, V. Gui, F. Alexa, and C. Toma, "Fast and accurate background subtraction for video surveillance, using an adaptive mode -tracking algorithm," in Proceedings of the 2005 WSEAS international conference on Dynamical systems and control, ser. CONTROL'05. Stevens Point, Wisconsin, USA: World Scientific and Engineering Academy and Society (WSEAS), 2005, pp. 391-397.
[12] O. Barnich and M. V. Droogenbroeck, "Vibe: A universal background subtraction algorithm for video sequences," IEEE Transactions on Image Processing, vol. 20, no. 6, pp. 17091724, 2011.

[13] X. Ding and L. Carin, "Separating background and foregroundin video based on a nonparametric bayesian model," in IEEE Workshop on statistical signal processing (SSP), Nice, June 2011, pp. 321-324.

[14] L. Cheng, M. Gong, D. Schuurmans, and T. Caelli, "Real-time discriminative background subtraction," IEEE Transactions on Image Processing, vol. 20, no. 5, pp. 1401-1414, 2011.

[15] B. Zhang, Y. Gao, S. Zhao, and B. Zhong, "Kernel similarity modeling of texture pattern flow for motion detection in complex background," IEEE Transactions on Circuits and Systems for Video Technology, vol. 21, no. 1, pp. 29-38, 2011.

[16] S. Zhang, H. Yao, and S. Liu, "Spatial-temporal nonparametric background subtraction in dynamic scenes," in ICME. IEEE, 2009, pp. 518-521.

[17] G. Dalley, J. Migdal, and W. E. L. Grimson, "Background subtraction for temporally irregular dynamic textures," in $W A C V, 2008$, pp. 1-7.

[18] Y. Sheikh and M. Shah, "Bayesian modeling of dynamic scenes for object detection," IEEE Transactions on Pattern Analysis and Machine Intelligence, vol. 27, no. 11, pp. 17781792, Nov. 2005.

[19] L. Li, W. Huang, I. Y. H. Gu, and Q. Tian, "Statistical modeling of complex backgrounds for foreground object detection," IEEE Transactions on Image Processing, vol. 13, no. 11, pp. 1459-1472, 2004.

[20] B. L. Xie, V. Ramesh, and T. E. Boult, "Sudden illumination change detection using order consistency," Image and Vision Computing, vol. 22, no. 2, pp. 117-125, Feb. 2004.

[21] Y. Ren, C. S. Chua, and Y. K. Ho, "Statistical background modeling for non-stationary camera," Pattern Recognition Letters, vol. 24, no. 1-3, pp. 183-196, Jan. 2003.

[22] J. Zhong and S. Sclaroff, "Segmenting foreground objects from a dynamic textured background via a robust kalman filter," in ICCV, 2003, pp. 44-50.

[23] A. Hyvaerinen and E. Oja, "Independent component analysis: algorithms and applications," Neural Networks, vol. 13, no. 4-5, pp. 411-430, 2000.

[24] D. M. Tsai and S. C. Lai, "Independent component analysisbased background subtraction for indoor surveillance," IEEE Transactions on Image Processing, vol. 18, no. 1, pp. 158167, Jan. 2009.

[25] C. Jutten and J. Herault, "Blind separation of sources, part I: An adaptive algorithm based on neuromimetic architecture," Signal Processing, vol. 24, pp. 1-10, 1991.

[26] V. Caselles, R. Kimmel, and G. Sapiro, "Geodesic active contours," International Journal of Computer Vision, vol. 22, pp. 61-79, 1995. 
[27] J. A. Sethian, Level Set Methods and Fast Marching Methods. Cambridge University Press, 1999.

[28] E. Durucan and T. Ebrahimi, "Change detection and background extraction by linear algebra," Proceedings of IEEE, vol. 89, no. 10, pp. 1368-1381, Oct. 2001.

[29] "Wallflower Dataset," http://research.microsoft.com/en-us/ um/people/jckrumm/wallflower/testimages.htm.

[30] B. White and M. Shah, "Automatically tuning background subtraction parameters using particle swarm optimization," in ICME. IEEE, 2007, pp. 1826-1829.

[31] N. A. Setiawan, S.-J. Hong, J.-W. Kim, and C.-W. Lee, "Gaussian mixture model in improved HLS color space for human silhouette extraction," in ICAT, ser. Lecture Notes in Computer Science, Z. Pan, A. D. Cheok, M. Haller, R. W. H. Lau, H. Saito, and R. Liang, Eds., vol. 4282. Springer, 2006, pp. $732-741$.

[32] H. Wang and D. Suter, "A re-evaluation of mixture of gaussian background modeling [video signal processing applications]," in Acoustics, Speech, and Signal Processing, 2005. Proceedings. (ICASSP '05). IEEE International Conference on, vol. 2, march 2005, pp. ii/1017 - ii/1020 Vol. 2.

[33] A. P. Pentland, B. Rosario, and N. M. Oliver, "A bayesian computer vision system for modeling human interactions," in CVS, 1999, p. 255 ff.

[34] S. S. Bucak, B. Günsel, and O. Gursoy, "Incremental nonnegative matrix factorization for dynamic background modelling," in PRIS, A. L. N. Fred and A. K. Jain, Eds. INSTICC PRESS, 2007, pp. 107-116.

[35] X. Li, W. Hu, Z. Zhang, and X. Zhang, "Robust foreground segmentation based on two effective background models," in Multimedia Information Retrieval, M. S. Lew, A. D. Bimbo, and E. M. Bakker, Eds. ACM, 2008, pp. 223-228. 